Mineralogical Journal, Vol. 5, No. 4, PP. 239-248, Mar., 1968

\title{
EFFECT OF PARTICLE SIZE ON ABSORPTION OF X-RAYS REFLECTED FROM MIXED POWDER (II) EXTRA ABSORPTION COEFFICIENT
}

\author{
MASAJI SHIMAZU* \\ Research Laboratory, Nihon Cement Co., Koto-ku, Tokyo \\ and \\ SUKEAKI HOSOYA** \\ Institute for Solid State Physics, University of Tokyo, \\ Azabu, Minato-ku, Tokyo
}

\begin{abstract}
In the previous paper (Part I), the 'self-absorption factor, $\tau_{1}$ ' ' and the ' extra absorption coefficient, $\Delta \mu$,' were derived and introduced to the formula of reflected X-ray intensity. In this paper, the coefficient $\Delta \mu$ is proved to be non-negative in a mixed powder of multiple components and to be zero in a powder of single component. The relation of magnitudes between $\Delta \mu / \mu$ and $\mu D$ is estimated on the assumption of $\mu>\left(\mu_{i} \sim \mu_{j}\right)$.

Discussion is made on the magnitude of particle size $D$ to make the particle effects on reflected X-ray intensity negligible.
\end{abstract}

\section{Introduction}

When particle sizes of a homogeneously mixed powder are sufficiently small and the powder is densely packed, the X-ray intensity reflected from the powder block having a flat surface can be given by a simple formula as (9) in this paper. In the case of coarser particles, however, the formula must be corrected by some factors.

* Present address: National Institute for Researches in Inorganic Materials, 28-60, Honkomagome 2 chome, Bunkyo-ku, Tokyo.

** The contribution by this author is limited to the proof of non-negativity of $\Delta \mu$. 
In Part I of this paper, two factors, the self-absorption factor $\tau_{1}$ and the extra absorption coefficient $\Delta \mu$, were theoretically derived. The former is due to the absorption by the particles causing X-ray reflection and the latter is due to the absorption in case a powder has multiple components. It was there assumed that the variation of a true absorbing path length is negligible among many $\mathrm{X}$-ray beams reflected from layer elements of a specimen at a level of the same depth, and that so-called 'extinction effects' are out of scope in the course of study, though those are not always negligible.

The reflected X-ray intensity, $I_{1}$, of the first component in a mixed powder composed of $n$ kinds of components was thus given by

$$
\begin{aligned}
I_{1} & =K_{1} \cdot \tau_{1} \cdot \frac{1}{1-(\Delta \mu / \mu)} \frac{f_{1}}{\mu} \\
& =K_{1} \cdot \tau_{1} \cdot \frac{1}{1-(\Delta \mu / \mu)} \frac{x_{1} / \rho_{1}}{x_{1}\left(\mu_{1}^{*}-\mu_{M}^{*}\right)+\mu_{M}^{*}},
\end{aligned}
$$

where

$$
\begin{aligned}
& \frac{f_{1}}{\mu}=\frac{\rho\left(x_{1} / \rho_{1}\right)}{\mu}=\frac{x_{1} / \rho_{1}}{\mu^{*}}, \\
& \Delta \mu=\frac{1}{2} H\left(\sum_{i=1}^{n} f_{i} \mu_{i}{ }^{2}-\mu^{2}\right),
\end{aligned}
$$

or

and

$$
\begin{gathered}
\frac{\Delta \mu}{\mu}=\frac{1}{2}\left(\rho^{\prime} / \rho\right)^{1 / 3} \mu D \frac{\sum_{i=1}^{n} f_{i} \mu_{i}{ }^{2}-\mu^{2}}{\mu^{2}}, \\
H=\left(\rho^{\prime} / \rho\right)^{1 / 3} D, \\
=0.9047 D, \\
f_{i}=\frac{x_{i} / \rho_{i}}{\sum_{i=1}^{n}\left(x_{i} / \rho_{i}\right)}, \quad x_{i}=\frac{f_{i} \rho_{i}}{\sum_{i=1}^{n} f_{i} \rho_{i}},
\end{gathered}
$$

$$
\tau_{1}=\frac{1}{V_{0}} \int_{0}^{V_{0}} \exp \left(-\mu_{1} z\right) \cdot d V_{0}
$$


The value of $0.9047 D$ was derived assuming that the particles are spherical with an equal diameter $D$ (i.e., mean particle size of the powder) and packed in the way of closest packing. A weight fraction $x_{i}$ is interchangeable with the volume fraction $f_{i}$ by (6). The physical meaning of the above formulae and the definition of symbols were described in Part 1 , and the symbols will be used in common in this paper.

In (1), the correction terms correspond to

$$
\tau_{1} \cdot \frac{1}{1-(\Delta \mu / \mu)} \cdot
$$

If $1-\tau_{1}$ and $\Delta \mu / \mu$ are sufficiently small, the magnitude of (8) approaches to unity, and then the reflected intensity can be given by

$$
I_{1}^{\prime}=K_{1}\left(f_{1} / \mu\right) \text {. }
$$

Both $1-\tau_{1}$ and $\Delta \mu / \mu$ are negligible, if the magnitude of $\mu_{1} D_{1}$ and $\mu D$ makes the reflected intensity fall in the range of an allowable error. This relation will be discussed in the following chapters.

\section{The sign of $\Delta \mu$}

An elementary proof of non-negativity of $\Delta_{\mu}$ is as follows:

$$
\begin{aligned}
Y & \equiv \sum_{i=1}^{n} f_{i} \mu_{i}{ }^{2}-\mu^{2}=\sum_{i=1}^{n} f_{i} \mu_{i}{ }^{2}-\left(\sum_{i=1}^{n} f_{i} \mu_{i}\right)^{2} \\
& =\sum_{i=1}^{n} f_{i} \mu_{i}{ }^{2}-\sum_{i=1}^{n} f_{i}{ }^{2} \mu_{i}{ }^{2}-2 \sum_{\substack{i=1 \\
i<j}}^{n} f_{i} f_{j} \mu_{i} \mu_{j} \\
& =\sum_{i=1}^{n} f_{i}\left(1-f_{i}\right) \mu_{i}{ }^{2}-2 \sum_{\substack{i=1 \\
i<j}}^{n} f_{i} f_{j} \mu_{i} \mu_{j} \\
& =\sum_{i=1}^{n} f_{i}\left(\sum_{i=1}^{n} f_{i}-f_{i}\right) \mu_{i}{ }^{2}-2 \sum_{i=1}^{n} f_{i} f_{j} \mu_{i} \mu_{j} \\
& =\sum_{i=1}^{n} f_{i} f_{j}\left(\mu_{i}-\mu_{j}\right)^{2} \geqq 0,
\end{aligned}
$$


because of $f_{i}>0$, and $f_{j}>0$. On the other hand, $H>0$. Therefore

$$
\Delta \mu \equiv \frac{1}{2} H Y=\frac{1}{2} H\left(\sum_{i=1}^{n} f_{i} \mu_{i}^{2}-\mu^{2}\right) \geqq 0,
$$

where the equality is satisfied in the trivial case of single component. Apart from that case, it is valid only when all $\mu_{i}$ 's are the same, though it does not seem likely to occur.

It seems worthwhile to mention that the above non-negativity relation is included in a more general theorem on the inequality. According to this theorem, the average value, $\overline{\varphi(\mu)}$, of any continuous. convex function, $\varphi(\mu)$, is not smaller than $\varphi(\bar{\mu})$ where $\bar{\mu}$ is the average value of $\mu$. The present case corresponds to a special case when $\varphi(\mu)=\mu^{2}$.

\section{Relation between $\Delta \mu / \mu$ and $\mu D$}

The object of this chapter is to estimate the magnitude of $\mu D$ to make $\Delta \mu / \mu$ negligible, accordingly, to make $1 /[1-(\Delta \mu / \mu)]$ approach unity. When the volume fractions $f_{i}$ 's (or weight fractions $x_{i}$ 's) and the linear absorption coefficients $\mu_{i}$ 's are known, the magnitude of $\Delta \mu / \mu$ can be determined by the formula

$$
\frac{\Delta \mu}{\mu}=0.4524 \mu D \frac{\sum_{i=1}^{n} f_{i} \mu_{i}^{2}-\mu^{2}}{\mu^{2}},
$$

which is obtained from (3) and (5). However, if the magnitudes of $f_{i}$ 's and $\mu_{i}$ 's are known, the quantitative $\mathrm{X}$-ray analysis to which the subject of this paper is related is no longer necessary.

Thus, we must here consider a general case. We must treat this problem with making some assumptions, because the upper and the lower limits are unknown for the values of $\mu_{i}$ 's in (12).

It is necessary that another expression without including $f_{i}$ 's and $\mu_{i}$ 's should be substituted for the term $\left(\sum_{i=1}^{n} f_{i} \mu_{i}{ }^{2}-\mu^{2}\right) / \mu^{2}$, in order to. obtain the relation between $\Delta \mu / \mu$ and $\mu D$. 
Now, assume that the difference $\mu_{i} \sim \mu_{j}$ is not larger than $\mu$. Then, the relation

$$
\mu^{2} \sum_{\substack{i=1 \\ i<j}}^{n} f_{i} f_{j}>\sum_{\substack{i=1 \\ i<j}}^{n} f_{i} f_{j}\left(\mu_{i}-\mu_{j}\right)^{2}
$$

clearly holds. Here, let the arithmetic mean value of $f_{i}$ 's be $f_{0}(=1 / n)$, and the deviation of $f_{i}$ from $f_{0}$ be $\varepsilon_{i}$, namely,

$$
f_{i}-f_{0} \equiv f_{i}-\frac{1}{n}=\varepsilon_{i},
$$

where $\sum_{i=1}^{n} f_{i}=1$ and $n$ is an integer $(n \geqq 2)$ which is the number of kinds of components. Then, the following equation can be derived:

$$
1=\left(\sum_{i=1}^{n} f_{i}\right)^{2}=\sum_{i=1}^{n} f_{i}^{2}+2 \sum_{\substack{i=1 \\ i<j}}^{n} f_{i} f_{j},
$$

namely,

$$
\begin{aligned}
2 \sum_{\substack{i=1 \\
i<j}}^{n} f_{i} f_{j} & =1-\sum_{i=1}^{n} f_{i}{ }^{2}=1-\sum_{i=1}^{n}\left(\frac{1}{n}+\varepsilon_{i}\right)^{2} \\
& =1-\frac{1}{n}-\sum_{i=1}^{n} \varepsilon_{i}{ }^{2}
\end{aligned}
$$

because $\sum_{i=1}^{n} \varepsilon_{i}=0$. Therefore, the relation

$$
\frac{1}{2}\left(1-\frac{1}{n}\right)>\sum_{\substack{i=1 \\ i<j}}^{n} f_{i} f_{j}
$$

holds, and, from (10), (13) and (16), the relation

$$
\frac{1}{2}\left(1-\frac{1}{n}\right) \mu^{2}>\sum_{i=1}^{n} f_{i} \mu_{i}{ }^{2}-\mu^{2}
$$

also holds.

Consequently, from (12) and (17), we obtain the relation

$$
0.2262 \mu D\left(1-\frac{1}{n}\right)>\frac{\Delta \mu}{\mu},
$$


Besides, jwe have the relation

$$
1>\frac{\Delta \mu}{\mu} \geqq 0,
$$

because $\Delta \mu \geqq 0$ as shown in (11), and because $\mu>\Delta \mu(i . e ., \mu=\beta+\Delta \mu)$ where $\mu>0$ and $\beta>0$ as mentioned in p. 188 of Part I ( $\beta$ corresponds to the linear absorption coefficient of the residual path which excludes the 'reflecting-particle').

The value of $0.2262 \mu D(1-1 / n)$ is an upper limit of the magnitude of $\Delta \mu / \mu$ under consideration, and it must be less than 1 . Under the assumption of $\mu>\left(\mu_{i} \sim \mu_{j}\right)$, the magnitude of $\Delta \mu / \mu$ in practice is sufficiently smaller than $0.2262 \mu D(1-1 / n)$. In other words, in order to expect that the magnitude of $\Delta \mu / \mu$ is less than a definite value $(\Delta \mu / \mu)_{d}$, the value less than $(\Delta \mu / \mu)_{d} / 0.2262(1-1 / n)$ is safe enough for the condition of $\mu D$. This is represented by a formula

$$
\mu D \leqq 4.42 \frac{n}{n-1}\left(\frac{\Delta \mu}{\mu}\right)_{d} .
$$

Formula $(20)$ is not a necessary condition but a sufficient one, because the left side of (18) shows an upper limit of $\Delta \mu / \mu$. According to $(20)$, the magnitude of $\mu D$ decreases as the number $n$ of the kinds of components increases, when a certain magnitude $(\Delta \mu / \mu)_{d}$ is appointed. However, whether this tendency is valid or not in practice is unknown.

The treatment by means of the assumption of $\mu>\left(\mu_{i} \sim \mu_{j}\right)$ is applicable to many examples, for instance, to many of the mixtures of rock-forming minerals, or many of the ceramic materials in which $\mu_{i} \sim \mu_{j}$ are not so large. However, in practical cases, whether $\mu$ is larger than the difference $\mu_{i} \sim \mu_{j}$ or not is unknown before carrying out quantitative analysis, because $\mu\left(=\sum_{i=1}^{n} f_{i} \mu_{i}\right)$ and the values of $f_{i}$ 's are unknown.

Thus, we cannot but use the largest $\mu_{i}$ instead of $\mu$ in (20). Then, the magnitude derived from this modified formula may give a 
much severer condition for $\mu D$ or for the particle size $D$ so that it gives a sufficiently safe condition.

Relation between the relative error of reflected $X$-ray intensity and the particle size of a mixed powder.

Let us derive the mean particle size $D$ in a mixed powder small enough to make the relative error $\Delta I_{1}{ }^{\prime} / I_{1}{ }^{\prime}$ of reflected intensity negligible, when formula (9) is used instead of (1). The relative error $\Delta I_{1}{ }^{\prime} / I_{1}^{\prime}$ is given by

$$
\begin{aligned}
\frac{\Delta I_{1}^{\prime}}{I_{1}^{\prime}} & =\frac{K_{1}\left(f_{1} / \mu\right)-K_{1}\left(f_{1} / \mu\right) \tau_{1}[1 /(1-\Delta \mu / \mu)]}{K_{1}\left(f_{1} / \mu\right)} \\
& =1-\tau_{1} \frac{1}{1-(\Delta \mu / \mu)} .
\end{aligned}
$$

Formula (21) can be re-written as (22) with the parameters $b$ and $c$ as follows :

$$
\begin{aligned}
\frac{\Delta I_{1}^{\prime}}{I_{1}^{\prime}} & =1-(1-b)(1+c) \\
& =b-c+b c,
\end{aligned}
$$

where

$$
\begin{gathered}
b=1-\tau_{1}, \\
1>b>0,
\end{gathered}
$$

because $\tau_{1}$ is the function with the range of $1>\tau_{1}>0$ as already mentioned in p. 190 of Part I, and

$$
\begin{gathered}
c=\frac{1}{1-(\Delta \mu / \mu)}-1, \\
c \geqq 0,
\end{gathered}
$$

because of (19).

If the values $b$ and $c$ are as small as negligible, (9) can be used 
instead of (1). The values $b$ and $-c$ have opposite signs and both are so small that $b c$ may be negligible in comparison with $b$ and $c$, and therefore, the smaller $D$ among two upper limits of $D$ values which are derived in order to suppress the values $b$ and $c$ under certain value should be chosen as the value of $D$.

Before considering the relation between particle size $D$ and the values of $b$ and $c$, the brief review of $\tau_{1}$ and $\Delta \mu / \mu$ may be necessary. $\tau_{1}$ is a monotonically decreasing function as $\mu_{1} D_{1}$ (or $D_{1}$ ) increases as already mentioned in p. 190 of Part I. On the other hand, $\Delta \mu / \mu$ is a monotonically increasing function as $D$ increases, as known from (3). Here, $\mu$ and $\mu_{1}$ are the linear absorption coefficient of a mixture and the linear absorption coefficient of the first component (which causes $\mathrm{X}$-ray reflection) respectively. $D$ and $D_{1}$ are the mean particle size of the mixture and the mean particle size of the first component respectively.

The effect of $\tau_{1}$ causes attenuation of the reflected intensity $I_{1}$ $\left(=I_{1}{ }^{\prime}-\Delta I_{1}{ }^{\prime}\right.$ ) as particle size $D_{1}$ increases (see Part I); in other words, the value $b$ increases as $D_{1}$ increases (also see (23)). On the other hand, the effect of $\Delta \mu$ causes augmentation (recovery) of $I_{1}$ as particle size $D$ increases; in other words, the value $-c$ decreases as $D$ increases (see (3) \& (25)). Thus the effects on reflected intensity $I_{1}$ by $\tau_{1}$ and $\Delta \mu$ have a tendency to counterbalance each other, in other words, the values $b$ and $-c$ have this tendency on relative error $\Delta I_{1}{ }^{\prime} / I_{1}^{\prime}$ as the particle size increases.

Now, let us consider the particle size to make $\Delta I_{1}{ }^{1} / I_{1}{ }^{\prime}$ negligible. This should be obtained from the relation between $\tau_{1}$ and $\mu_{1} D_{1}$ and from the relation between $\Delta \mu / \mu$ and $\mu D$. The relation $\tau_{1}$ and $\mu_{1} D_{1}$ can be obtained by direct reference to the numerical tables by Evans and Ekstein (1952). Accordingly, the relation between $b$ and $\mu_{1} D_{1}$ can also be derived from their tables and (23). On the other hand, though $\mu>\left(\mu_{i} \sim \mu_{f}\right)$ should be assumed, the relation between $\Delta \mu / \mu$ and $\mu D$, accordingly, the relation between $c$ and $\mu D$ can be calculated from 
(20) and (25) as follows:

$$
\mu D \leqq 4.42 \frac{n}{n-1} \frac{\gamma}{1+\gamma},
$$

where $\gamma$ is a certain value of $c$. In other words, in order to expect that the magnitude of $c$ is less than a definite value $\gamma$, the value of the right-hand side of (27) is safe enough for the condition of $\mu D$. In (27), the relation $n /(n-1)<1$ holds, because $\lim _{n \rightarrow \infty} n /(n-1)=1$. Formula (27) as well as (20) is not a necessary condition but a sufficient one for the value of $\mu D$.

As far as the practical example of a mixed powder is concerned, $D_{1}$ may be unable to be measured though $D$ can be measured, and therefore $D$ must be substituted approximately for $D_{1} ; \mu_{1}$ can be calculated from the ideal chemical formula and ideal density of the first component, while $\mu$ cannot be calculated before carrying out quantitative analysis. Therefore, $\mu_{1} D$ should be substituted for $\mu_{1} D_{1}$ when Evans and Ekstein's table is applied, and $\mu_{l} D$ (where $\mu_{l}$ is the largest $\mu_{i}$ ) should be substituted for $\mu D$ when (27) is used. Thus, the smaller $D$ should be chosen as the value of $D$, in which the smaller $D$ can be derived from two upper limits of $D$ in order to suppress the values of $b$ and $c$ under a certain value.

When $\Delta I_{1}{ }^{\prime} / I_{1}{ }^{\prime} \leqq 0.1$ is required, for example, it is safe enough that both $b$ and $c$ are less than 0.1. According to Evans and Ekstein's table, the condition $\mu_{1} D \leqq 0.1$ is necessary, while according to (27), $\mu_{l} D \leqq 0.4<0.4 n /(n-1)$ is necessary. Thus, in this case, formula (9) can be used instead of (1) provided $\mu_{1} D \leqq 0.1$ and $\mu_{l} D \leqq 0.4$ are satisfied. The smaller $D$, which is deduced from these two upper limits, should be chosen as the value of $D$.

\section{Summary and conclusion}

The intensity of X-rays reflected from a mixed powder can be given by (1), in which the self-absorption factor $\tau_{1}$ and the extra 
absorption coefficient $\Delta \mu$ are involved as the correction terms for the particle effects of absorption.

The self-absorption factor is always positive, less than unity in both powders of single and multiple components, and the factor is a monotonically decreasing function with the increase of particle size (see p. 190 of Part I).

The extra absorption coefficient is non-negative in the case of multiple components and it is zero in the case of single component (see (11)). The $\Delta \mu / \mu$ is less than unity and it is an increasing function with the increase of particle size, as shown in (3) and (19).

The effect of the self-absorption factor causes the attenuation of reflected X-ray intensity (see Part I), while the effect of the extra absorption coefficient causes the augmentation (see (1), (11) \& (19)). Therefore, both effects have a tendency to counterbalance each other.

If (9) is used instead of (1), the relative error of reflected intensity is given by (21) or (22). The smaller $D$, which can be derived from two upper limits of $D$ values in order to suppress the values $b$ and $c$ under a certain value, should be chosen as the required value of $D$.

One of two upper limits of $D$ can be obtained by (7) (see Part I) and (23), and the other is obtained as an order of magnitude by (27) in which the largest $\mu_{i}$ should be used instead of $\mu$ in (27), and formula (27) has been derived under the assumption of $\mu>\left(\mu_{i} \sim \mu_{j}\right)$.

\section{REFERENCES}

Evans, H. T., \& Ekstein, M. G. (1952). Acta Cryst., 5, 540-542.

Shimazu, M. (1967). Miner. J., 5, 180-198. (Part I of this paper).

Manuscript received 25 August 1967. 